|IIIIIIIIIIIIIIIIIIIIIIIIIIIIIIIIIII

Original Article

IIIIIIIIIIIIIIIIIIIIIIIIIIIIIIIIII

\title{
Bioconversion of 5-deoxystrigol stereoisomers to monohydroxylated strigolactones by plants
}

\author{
Kotomi Ueno, "Hitomi Nakashima, Masaharu Mizutani, \\ Hirosato TAKikawa and Yukihiro Sugimoto* \\ Graduate School of Agricultural Science, Kobe University, Nada, Kobe 657-8501, Japan
}

(Received March 29, 2018; Accepted June 23, 2018)

\begin{abstract}
The bioconversion of 5-deoxystrigol (5DS) and 4-deoxyorobanchol (4DO), the simplest canonical strigolactones (SLs), into monohydroxylated SLs such as strigol, sorgomol and orobanchol was confirmed by administering of stable isotope-labeled substrates to hydroponically grown plants. Liquid chromatography-mass spectrometry analyses established that 5DS was stereoselectively converted into strigol and sorgomol by cotton (Gossypium hirsutum) and Chinese milk vetch (Astragalus sinicus), respectively. 4DO was converted into orobanchol by rice (Oryza sativa). However, the red bell pepper (Capsicum annuum), red clover (Trifolium pratense), and pea (Pisum sativum) negligibly converted 4DO into orobanchol. The red bell pepper converted ent-4DO into 2',8-bisepi-sorgomol. These results suggest that some plants generate orobanchol without passing through 4DO. (C) Pesticide Science Society of Japan

Keywords: strigolactones, 5-deoxystrigol, hydroxylation, stereoselective, biosynthesis.
\end{abstract}

Electronic supplementary material: The online version of this article contains supplementary material (Supplemental Fig. S1S4), which is available at http://www.jstage.jst.go.jp/browse/jpestics/

\section{Introduction}

Strigolactones (SLs) are important rhizosphere signaling molecules that induce hyphal branching of arbuscular mycorrhizal (AM) fungi, an essential step in establishing symbiosis, ${ }^{1)}$ and trigger the seed germination of root parasitic weeds such as witchweed (Striga spp.) and broomrapes (Orobanche and Phelipanche spp.). ${ }^{2,3)}$ SLs act as a novel class of plant hormones that regulate shoot branching and root development. ${ }^{3-5)}$ These molecules are present in liverworts and Charales, which suggests that SLs appeared in the streptophytes to control rhizoid elongation rather than to promote AM symbiosis. $\left.{ }^{6}\right)$

To date, more than 10 naturally occurring SLs have been elucidated with regard to their structure with an absolute configuration. ${ }^{7)}$ Canonical SLs consist of a tricyclic lactone (ABC rings) and a methylfuranone moiety (the $\mathrm{D}$ ring) connected through an enol ether linkage, although avenaol ${ }^{8)}$ and heliolactone, ${ }^{9)}$ whose $\mathrm{B}$ and $\mathrm{C}$ rings are unclosed, have been isolated. The simplest canonical SLs are 5-deoxystrigol (5DS, 1) and its diastereomer, 4-deoxyorobanchol (4DO, 2), namely deoxy-SLs.

\footnotetext{
* To whom correspondence should be addressed.

E-mail: yukihiro@kobe-u.ac.jp

\# Present address: Faculty of Agriculture, Tottori University, Koyama, Tottori, 680-8553, Japan.

Published online August 1, 2018

(c) Pesticide Science Society of Japan
}

Modifying deoxy-SLs through hydroxylation, acetylation, oxidation, or decarboxylation can generate the variety of canonical SL structures occurring in nature. Different species and different cultivars in one crop species produce qualitatively and quantitatively different SL mixtures. ${ }^{7,10,11)}$ These differences are likely to be critical for triggering interactions with rhizospheric organisms. SL-deficient pea and tomato mutants decreased the colonization of AM fungi as compared to the wild types. ${ }^{4,12)}$ Strigasusceptible sorghum and maize exuded the deoxy-SL 5DS (1), while Striga-resistant cultivars exuded mainly monohydroxylated SL sorgomol (3). ${ }^{13,14)}$

Hydroxy-SLs (HO-SLs) such as strigol (4), sorgomol (3) and orobanchol (5) have been found in the root exudates of various plants. Strigol (4) was identified as the first natural Striga germination stimulant from cotton (Gossypium hirsutum L.). ${ }^{15)}$ Strigol (4) has the hydroxyl group at C-5 in the A ring. Sorgomol (3), which was isolated from sorghum (Sorghum bicolor (L.) Moench) root exudates, ${ }^{16)}$ has the hydroxyl group at C-9 in the A ring. Alectrol (6), which was isolated from the cowpea (Vigna unguiculata (L.) Walp), was initially presumed as an isomer of strigol (4), ${ }^{17)}$ but the compound was unambiguously established to be acetylated orobanchol. ${ }^{18)}$ Orobanchol (5) was isolated from the root exudates of red clover (Trifolium pratense L.), ${ }^{19)}$ rice (Oryza sativa L.), ${ }^{10)}$ and many other plant species. ${ }^{7)}$ Orobanchol (5) has the hydroxyl group at C-4 in the B ring. All canonical SLs identified so far, including HO-SLs, have the $R$ configuration at $\mathrm{C}-2^{\prime}$ in the $\mathrm{D}$ ring, whereas the stereochemistry of the $\mathrm{B}-\mathrm{C}$ 


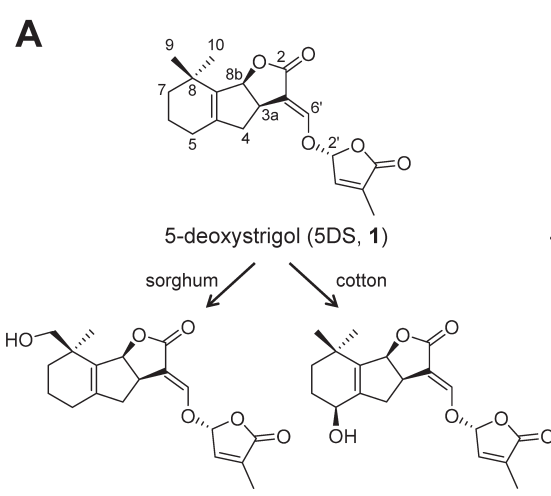

sorgomol (3)

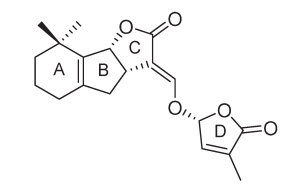

4-deoxyorobanchol (4DO, 2)

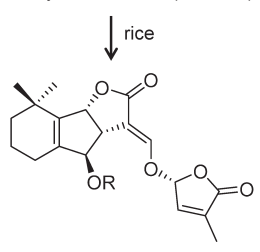

$\mathrm{R}=\mathrm{H}$ : orobanchol $(\mathbf{5})$ $\mathrm{R}=\mathrm{Ac}$ : alectrol $(6)$
B

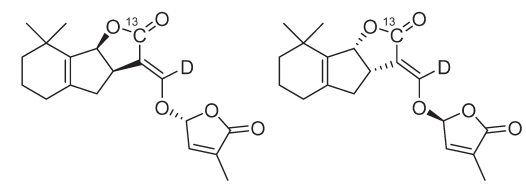

1a $\left(\left[2-{ }^{13} \mathrm{C}, 6^{\prime}-\mathrm{D}\right]-5 \mathrm{DS}\right)$

1b (ent-1a)

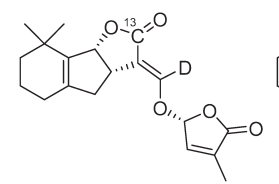

2a $\left(\left[2-{ }^{13} \mathrm{C}, 6^{\prime}-\mathrm{D}\right]-4 \mathrm{DO}\right)$

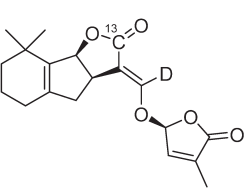

2b (ent-2a)
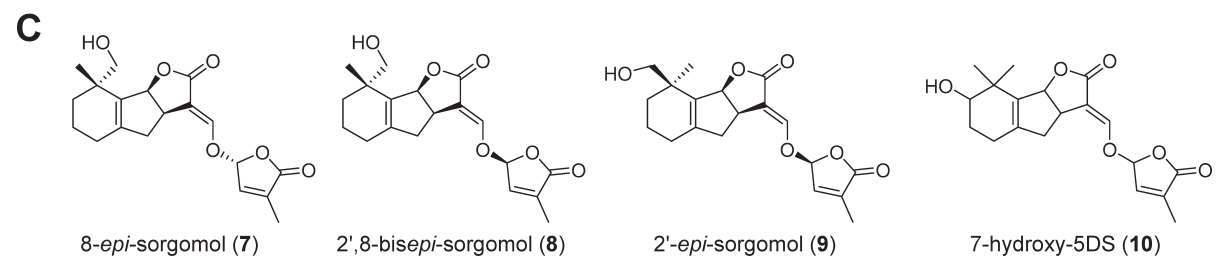

Fig. 1. Strigolactone structures. (A) Bioconversion of deoxy-SLs (1 and 2) into hydroxy-SLs (3-5) by plants. ${ }^{11,20,21)}$ (B) Structures of $\left[2-{ }^{13} \mathrm{C}, 66^{\prime}-\mathrm{D}\right]-5 \mathrm{DS}(\mathbf{1} \mathbf{a}$ and $\mathbf{1 b}$ ) and $\left[2-{ }^{13} \mathrm{C}, 6^{\prime}-\mathrm{D}\right]-4 \mathrm{DO}(\mathbf{2} \mathbf{a}$ and $\mathbf{2 b}$ ) stereoisomers. (C) Structures of sorgomol isomers 7-9 and 7-hydroxy-5DS (10). Compound $\mathbf{1 0}$ is a mixture of racemic diastereomers.

junction (at C-3a and C-8b) was variable. Strigol (4) and sorgomol (3) have the $(8 \mathrm{bS})$ configuration, while orobanchol (5) has the $(8 \mathrm{~b} R)$ configuration. Although synthetic HO-SLs theoretically have eight stereoisomers, each naturally occurring HO-SL has only one stereoisomer.

Based on the structural similarity, the simplest deoxy-SLs are thought to be precursors for HO-SLs; that is, 5DS (1) can be converted into sorgomol (3) or strigol (4), and 4DO (2) can be converted into orobanchol (5) (Fig. 1A). To confirm the conversion of 5DS (1) into sorgomol (3), we administered deuterium-labeled 5DS $\left(d_{1}-1\right)$ to hydroponically cultured sorghum and detected labeled sorgomol $\left(d_{1}-3\right)$ in the root exudates. ${ }^{11)}$ The conversion of 4DO (2) into orobanchol (5) was confirmed by in vitro experiments using rice CYP711 (Os1400). ${ }^{20)}$ However, Iseki et al. ${ }^{21)}$ reported that the cowpea was not able to convert $4 \mathrm{DO}$ (2) into orobanchol (5). They also demonstrated that cotton converted 5DS (1) into strigol (4), while the root culture of moonseed that produces strigol (4) failed to convert it.

The aim of our study was to demonstrate the conversion of deoxy-SLs into HO-SLs in various plant species to scrutinize the biosynthetic pathway of HO-SLs. We prepared ${ }^{13} \mathrm{C}$ - and deuterium-labeled 5DS stereoisomers and analyzed the root exudates of 12 plant species using liquid chromatography coupled with tandem mass spectrometry (LC-MS/MS) to select plants for administration experiments. We then administered stable isotopelabeled substrates to hydroponically cultured plants and monitored the metabolite production. Additionally, we discussed the validity of the biosynthetic pathway of HO-SLs.

\section{Materials and Methods}

\section{General}

Proton nuclear magnetic resonance $\left({ }^{1} \mathrm{H}\right.$ NMR) spectra were recorded in $\mathrm{CDCl}_{3}$ with a JNM-AL 300 spectrometer (JEOL, Tokyo, Japan) using tetramethylsilane as an internal standard, and chemical shifts are shown in ppm $(\delta)$. High-resolution electron impact mass spectra (HREIMS) were obtained on a JMS700 spectrometer (JEOL), whereas circular dichroism (CD) spectra were recorded with a J-805 spectropolarimeter (JASCO, Tokyo, Japan). LC/MS analyses were performed using an LC/ MS system (Waters, Milford, MA, USA) consisting of an Acquity Ultra Performance liquid chromatograph (UPLC) and an Acquity quadruple tandem mass spectrometer (TQ Detector). Data acquisition and analyses were performed using MassLynx 4.1 software (Waters).

\section{Chemicals}

Fluridone, uniconazole-P, and prohexadione were purchased from Wako (Osaka, Japan). $\mathrm{DCOOCH}_{3}$ (98+at\% D) and $\left[1-{ }^{13} \mathrm{C}\right]$-ethyl bromoacetate (99\%) were purchased from ACROS (Geel, Belgium) and CIL (Andover, MA, USA), respectively. Racemic [6'-D]-5DS isomers $\left(r a c-d_{1}-1\right.$ and $\left.r a c-d_{1}-2\right)$ and sorgomol isomers, 8-epi-sorgomol (7), 2',8-bisepi-sorgomol (8), 2'-episorgomol (9), and 7-hydroxy-5DS (10), were prepared using the method reported previously. ${ }^{11,22)}$ Racemic $\left[2-{ }^{13} \mathrm{C}, 6^{\prime}-\mathrm{D}\right]-5 \mathrm{DS}$ $(\mathbf{1 a} / \mathbf{1 b})$ and its diastereomer, $\left[2{ }^{13} \mathrm{C}, 6^{\prime}-\mathrm{D}\right]-4 \mathrm{DO}(\mathbf{2 a} / \mathbf{2 b})$, were prepared according to the method reported previously. ${ }^{23)}{ }^{1} \mathrm{H}$ NMR spectra of compounds $\mathbf{1 a} / \mathbf{1} \mathbf{b}$ and $\mathbf{2} \mathbf{a} / \mathbf{2} \mathbf{b}$ were identical to those of [6 $\left.6^{\prime}-\mathrm{D}\right]-5 \mathrm{DS}\left(d_{1}-1\right)$ and $\left[6^{\prime}-\mathrm{D}\right]-4 \mathrm{DO}\left(d_{1}-2\right)$, respec- 
tively. ${ }^{11)}$ HREIMS $m / z\left(\mathrm{M}^{+}\right)$: $\mathbf{1 a} / \mathbf{1 b}$, Calcd. for $\mathrm{C}_{18}{ }^{13} \mathrm{CH}_{21} \mathrm{DO}_{5}$ : 332.1564, Found: 332.1566; 2a/2b, Calcd. for $\mathrm{C}_{18}{ }^{13} \mathrm{CH}_{21} \mathrm{DO}_{5}$ : 332.1564, Found: 332.1568. The optical resolution of racemic $\left[2-{ }^{13} \mathrm{C}, 6^{\prime}-\mathrm{D}\right]-5 \mathrm{DS}(\mathbf{1} \mathbf{a} / \mathbf{1} \mathbf{b})$ was achieved by HPLC with a CHIRALPAK IA $(250 \times 10 \mathrm{~mm}, 5 \mu \mathrm{m})$ (Daicel Chemical, Osaka, Japan) using a mixed solvent of $30 \% \mathrm{EtOH}$ in $n$-hexane as the mobile phase at a flow rate of $3.0 \mathrm{~mL} / \mathrm{min}$. 5DS stereoisomers were detected by absorbance at $235 \mathrm{~nm}$. $\left[2-{ }^{13} \mathrm{C}, 6{ }^{\prime}-\mathrm{D}\right]$-ent-5DS (1b) and $\left[2-{ }^{13} \mathrm{C}, 6^{\prime}-\mathrm{D}\right]-5 \mathrm{DS}(\mathbf{1 a})$ were eluted at 11.2 and $17.1 \mathrm{~min}$, respectively. The optical resolution of racemic $\left[2-{ }^{13} \mathrm{C}, 6^{\prime}-\mathrm{D}\right]-$ $4 \mathrm{DO}(\mathbf{2} \mathbf{a} / \mathbf{2} \mathbf{b})$ was performed by HPLC with a CHIRALPAK IC $(250 \times 10 \mathrm{~mm}, 5 \mu \mathrm{m})$ using a mixed solvent of $37.5 \% \mathrm{EtOH}$ in $n$-hexane as the mobile phase at a flow rate of $3.0 \mathrm{~mL} / \mathrm{min}$. $\left[2-{ }^{13} \mathrm{C}, 6^{\prime}-\mathrm{D}\right]-4 \mathrm{DO}(\mathbf{2 a})$ and $\left[2-{ }^{13} \mathrm{C}, 6^{\prime}-\mathrm{D}\right]-$ ent-4DO (2b) were eluted at 16.9 and $19.9 \mathrm{~min}$, respectively. The absolute configuration of these isomers was determined on the basis of their CD Cotton effects in comparison with those reported previously. ${ }^{11)}$ The optical purity of all stereoisomers was more than 99.9\% ee, estimated by analytical chiral HPLC using the analytical columns. LC-ESI-MS/MS, parent ions of $\mathrm{m} / z$ 97: 1a, $\mathrm{m} / \mathrm{z}$ (base peak intensity \%), 332 (1.2), 333 (100.0), 334 (14.1), 335 (3.1); 1b, $m / z$ (base peak intensity \%), 332 (1.5), 333 (100.0), 334 (14.8), 335 (1.4); 2a, $m / z$ (base peak intensity \%), 332 (1.9), 333 (100.0), 334 (14.9), 335 (1.8); 2b, $m / z$ (base peak intensity \%), 332 (1.1), 333 (100.0), 334 (13.2), 335 (1.5).

\section{Plant material}

Seeds of the Chinese milk vetch (Astragalus sinicus L.), red clover (T. pratense L.), white clover (Trifolium repens L.), and sunflower (Helianthus annuus L. 'Hybrid sunflower') were generously supplied by Kaneko Seeds, Gunma, Japan. Rice (O. sativa, cv. Nipponbare) seeds were supplied by the National Institute of Agrobiological Sciences, Ibaraki, Japan. Tobacco (Nicotiana tabacum L., 'NA24') seeds were generously supplied by Professor Rossitza Batchvarova, AgroBioInstitute, Bulgaria. Cotton ( $G$. hirsutum L. 'Tonko' and G. arboreum L. 'Wawata') seeds were purchased from Cotton Bank Mashiko, Tochigi, Japan. Red bell pepper (Capsicum annuum L. 'grossum'), lettuce (Lactuca sativa L. 'Melbourne MT'), pea (Pisum sativum L. 'Hyogo kinusaya'), morning glory (Ipomoea nil (L.) Roth 'Kikyozaki kongo'), and tomato (Solanum lycopersicum L. 'Momotaro') seeds were obtained from a local supplier.

\section{Hydroponic culture}

Cotton seeds were surface-sterilized in a half-strength sodium hypochlorite solution for $15 \mathrm{~min}$ and rinsed thoroughly with tap water. The seeds were soaked in tap water, incubated for a day at $28^{\circ} \mathrm{C}$ in the dark, and then carefully husked. Seeds were plated on a wet Kimwipe in a glass petri dish and allowed to germinate for a day at $28^{\circ} \mathrm{C}$. Other plant seeds, except for purchased seeds, were surface-sterilized using $100 \% \mathrm{EtOH}$ for $5 \mathrm{~min}$. Seeds were plated on moistened filter papers (Advantec No.2) in a glass petri dish and incubated for 2 days at $20-25^{\circ} \mathrm{C}$ in the dark. Germinated seeds were transferred onto a mesh $(2.5 \mathrm{~mm}$,
$4.5 \times 4.5 \mathrm{~cm})$ set on a tap water-filled plastic cup $(150 \mathrm{~mL})$ with sealing by a slightly larger transparent plastic cup, and allowed to grow hydroponically in a phytotron under controlled conditions (light $18 \mathrm{hr}$ : dark $6 \mathrm{hr}$, at 23 or $28^{\circ} \mathrm{C}$ ). After a 6-day incubation, the sealing cup was removed, and the aquaculture water was exchanged for a nutrient solution (1/2000 HypoNeX or half-strength Hoagland's solution). The medium was exchanged every other day and plants were grown until roots were fully expanded in the cup. Before gathering root exudates, the medium was changed to tap water and incubated for at least 2 days. For qualitative analysis of SLs, aquaculture water was collected every 24 hr over 3 days.

\section{Extraction and analysis of root exudates}

Plants were incubated with tap water for $24 \mathrm{hr}$, and the aquaculture water filtered with a filter paper (Advantec No. 2) was passed through a NOBIAS RP-EX1 column (250 mg, $1.3 \mathrm{~mL}$ ) (Hitachi High-Technologies, Tokyo, Japan) that was washed with $\mathrm{MeOH}$ and equilibrated with distilled water $(10 \mathrm{~mL})$. The column was washed with water $(10 \mathrm{~mL})$, and the absorbed root exudates were eluted with $\mathrm{EtOH}$ and immediately evaporated in vacuo at $35^{\circ} \mathrm{C}$. The residue was dissolved in $30 \%$ EtOAc in $\mathrm{CH}_{2} \mathrm{Cl}_{2}(5 \mathrm{~mL})$ and passed through a silica gel column $(6 \mathrm{~mm} \times 1.5 \mathrm{~cm})$. The eluate was concentrated, dissolved in acetonitrile $(\mathrm{MeCN})$, and filtered through PTFE filter $(0.2 \mu \mathrm{m}$, $13 \mathrm{~mm}$, Waters). The filtrate was concentrated and redissolved in MeCN $(50 \mu \mathrm{L})$ to analyze an aliquot $(5 \mu \mathrm{L})$ using an LC/MS system. The UPLC analytical conditions were as follows: column, $100 \times 2.0 \mathrm{~mm}$ i.d., $2.5 \mu \mathrm{m}$, COSMOSIL Packed column $2.5 \mathrm{C}_{18^{-}}$ MS-II, (Nacalai Tesque, Inc., Kyoto, Japan), $30^{\circ} \mathrm{C}$; solvent, $50 \%$ (0-2 min), 50 to $70 \%$ (2-12 min, linear gradient), 70 to $100 \%$ (12-16 min, linear gradient) $\mathrm{MeOH}$ in $\mathrm{H}_{2} \mathrm{O}$; flow rate, $0.2 \mathrm{~mL}$ / min. The mass spectrometer was operated in positive electrospray ionization (ESI) mode with the capillary voltage at $3 \mathrm{kV}$, the source temperature at $120^{\circ} \mathrm{C}$, and the desolvation gas temperature at $350^{\circ} \mathrm{C}$. The nebulizer and desolvation $\mathrm{N}_{2}$ gas flows were 50 and $550 \mathrm{~L} / \mathrm{h}$, respectively. Fragmentation was performed by collision-induced dissociation with argon at $0.1 \mathrm{~mL} / \mathrm{min}$. The MRM transitions were set according to the mass spectra obtained from analyses of authentic compounds or molecular weights that were reported previously. ${ }^{24,25)}$ For searching known SLs, the transitions (channels) selected were at $\mathrm{m} / z$ 317>97 and $317>133$ for sorgolactone and sorgomol (3); 329>97 for strigol (4); $331>97$ and $331>216$ for 5 -DS (1) and 4-DO (2); $343>97$ for solanacol; 345>97 for oxo-SLs; 347>97 for HOSLs $\left([\mathrm{M}+\mathrm{H}]^{+}\right)$, mainly orobanchol (5), and alectrol (6); 347>233 for orobanchol (5) and alectrol (6); 361 >97 for heliolactone and methoxy-SLs; $364>317$ for HO-SLs $\left(\left[\mathrm{M}+\mathrm{NH}_{4}\right]^{+}\right)$, mainly sorgomol (3); 369>272 for HO-SLs $\left([\mathrm{M}+\mathrm{Na}]^{+}\right)$, mainly strigol (4); 389>97 and 389>233 for alectrol (6); 403>97 for 7-oxoalectrol; and 405>97 for fabacyl acetate. A parent ion scan was performed for the fragment ion at $m / z$ 97, which corresponds to the $\mathrm{D}$ ring, over the range of $50-450 \mathrm{~m} / z$, with a cone voltage of $30 \mathrm{~V}$ and a collision energy of $18 \mathrm{eV}$. 


\section{Administration of labeled 5DS stereoisomers}

Plants grown in HypoNeX or half-strength Hoagland's solution were transferred to tap water in the presence or absence of fluridone $(0.4 \mu \mathrm{M})$ after washing the roots with running water and incubated for 2 days in the phytotron. The culture medium was replaced by tap water $(150 \mathrm{~mL})$ with the indicated labeled 5DS isomers (100 ng) and allowed to metabolize the labeled substrate for $24 \mathrm{hr}$ in the presence or absence of fluridone $(0.4 \mu \mathrm{M})$. After the plants were removed, the aquaculture water was supplemented with 4-hydroxy-GR24 as an internal standard. The metabolites of the labeled compound (and endogenous SLs, when the plant was incubated without fluridone) were extracted, purified, and analyzed using the method described above. For confirmation of conversion by multiple reaction monitoring (MRM), the transitions selected were at $\mathrm{m} / z$ 315 $>97$ for 4-hydroxyGR24; $317>97$ for sorgomol (3); 318>97 for $d_{1}-3 ; 319>97$ for $\left[2-{ }^{13} \mathrm{C}, 66^{\prime}-\mathrm{D}\right]-3 ; 329>97$ for strigol $(4) ; 330>97$ for $d_{1}-4 ; 331>97$ for 5-DS (1), 4-DO (2), and $\left[2-{ }^{13} \mathrm{C}, 6{ }^{\prime}-\mathrm{D}\right]-4 ; 332>97$ for $d_{1}-1$ and $d_{1}-\mathbf{2} ; 333>97$ for $\left[2-{ }^{13} \mathrm{C}, 6^{\prime}-\mathrm{D}\right]-5-\mathrm{DS}$ isomers $(\mathbf{1 a}, \mathbf{1 b}, \mathbf{2} \mathbf{a}$, and 2b); 347>97 for HO-SLs $\left([\mathrm{M}+\mathrm{H}]^{+}\right)$, mainly orobanchol (5); $348>97$ for $d_{1}$-HO-SLs $\left([\mathrm{M}+\mathrm{H}]^{+}\right)$, mainly $d_{1}-5$; 349>97 for $\left[2-{ }^{13} \mathrm{C}, 66^{\prime}-\mathrm{D}\right]-\mathrm{HO}-\mathrm{SLs}([\mathrm{M}+\mathrm{H}]+)$, mainly $\left[2-{ }^{13} \mathrm{C}, 6^{\prime}-\mathrm{D}\right]-5$; $369>272$ for HO-SLs $\left([\mathrm{M}+\mathrm{Na}]^{+}\right)$, mainly strigol (4); 370>273 for $d_{1}$-HO-SLs $\left([\mathrm{M}+\mathrm{Na}]^{+}\right)$, mainly $d_{1}-4$; and $371>274$ for $\left[2-{ }^{13} \mathrm{C}, 6{ }^{\prime}-\mathrm{D}\right]-\mathrm{HO}-\mathrm{SLs}\left([\mathrm{M}+\mathrm{Na}]^{+}\right)$, mainly $\left[2-{ }^{13} \mathrm{C}, 6^{\prime}-\mathrm{D}\right]-4$. For the inhibition experiment, uniconazole-P $(0.1-10 \mu \mathrm{M})$ or prohexadione $(10 \mu \mathrm{M})$ was concomitantly added to the aquaculture water with the labeled substrate.

\section{Results}

1. Preparation of stable isotope-labeled 5-deoxystrigol stereoisomers

For administration experiments, we prepared stable isotopelabeled 5DS stereoisomers, $\left[2{ }^{13} \mathrm{C}, 6{ }^{\prime}-\mathrm{D}\right]-5 \mathrm{DS}$ (1a and $\left.\mathbf{1 b}\right)$ and $\left[2-{ }^{13} \mathrm{C}, 6^{\prime}-\mathrm{D}\right]-4 \mathrm{DO}$ (2a and 2b) (Fig. 1B), using almost the same method as reported previously. ${ }^{23)}$ The bicyclic enone was alkylated to the ${ }^{13} \mathrm{C}$-labeled oxo acid using ethyl bromoacetate (1$\left.{ }^{13} \mathrm{C}, 99 \%\right)$ by a two-step reaction. The oxo acid was reduced to obtain the ${ }^{13} \mathrm{C}$-labeled $\mathrm{ABC}$ ring. Formylation of the $\mathrm{ABC}$ ring with $\mathrm{DCO}_{2} \mathrm{Me}(98+$ at\% $\mathrm{D})$ and a subsequent coupling reaction with 5-bromo-3-methyl-2(5H)-furanone gave racemic $\left[2-{ }^{13} \mathrm{C}, 6^{\prime}-\mathrm{D}\right]-5 \mathrm{DS}(\mathbf{1} \mathbf{a} / \mathbf{1 b})$ and its diastereomer, namely racemic $\left[2-{ }^{13} \mathrm{C}, 6^{\prime}-\mathrm{D}\right]-4 \mathrm{DO}(\mathbf{2} \mathbf{a} / \mathbf{2} \mathbf{b})$. LC-MS/MS analysis of racemic compounds with a parent ion scan at $m / z 97$ showed the major precursor ion $[\mathrm{M}+\mathrm{H}]^{+}$at $m / z 333$, and the intensity of the ion at $m / z 331$, the $[\mathrm{M}+\mathrm{H}]^{+}$ion of unlabeled compounds, was under the detection limit. The racemic mixtures were optically separated with chiral HPLC to give four stereoisomers, $\left[2-{ }^{13} \mathrm{C}, 6^{\prime}-\mathrm{D}\right]-$ 5DS (1a), $\left[2-{ }^{13} \mathrm{C}, 6{ }^{\prime}-\mathrm{D}\right]-$ ent-5DS (1b), $\left[2-{ }^{13} \mathrm{C}, 6^{\prime}-\mathrm{D}\right]-4 \mathrm{DO}(\mathbf{2 a})$, and $\left[2-{ }^{13} \mathrm{C}, 6^{\prime}-\mathrm{D}\right]-$ ent $-4 \mathrm{DO}(\mathbf{2 b})$.

\section{Selection of highly monohydroxylated strigolactone-producing plants}

Twelve plant species, listed in Table 1, were grown hydroponically with a nutrient solution for 2-12 weeks and transferred into tap water to activate SL production. Root exudates in aquaculture filtrates were collected using a reversed-phase hydrophilic polymer. The root exudates that were absorbed to the polymer were eluted with EtOH and briefly purified via silica gel column chromatography for semi-quantitative analysis using LC-MS/ MS in MRM mode. Identification of known SLs was performed by comparing the retention times and peak intensities in each MRM channel with authentic compounds, 5DS (1), 4DO (2), sorgomol (3), strigol (4), orobanchol (5), alectrol (orobanchyl acetate, 6), 4-hydroxy-5DS, and sorgolactone. For instance, strigol (4) was detected by the channel at $\mathrm{m} / z$ 369 $>272$ with higher sensitivity than that at $m / z 347>97$, whereas orobanchol (5) was detected sensitively by the channel at $m / z$ 347>97 under

Table 1. Distribution of SL production by plants

\begin{tabular}{|c|c|c|c|c|c|}
\hline \multirow{2}{*}{ Plants } & \multirow{2}{*}{ Number of plants } & \multirow{2}{*}{ Growing period } & \multirow{2}{*}{$\begin{array}{l}\text { Concentration for } \\
\text { analysis }^{a)}\end{array}$} & \multicolumn{2}{|c|}{ Detected SLs } \\
\hline & & & & Major & Others \\
\hline Cotton (G. hirsutum) & 1 & 15 days & 2520 & 4 & 1 \\
\hline Cotton (G. arboreum) & 1 & 14 days & 2800 & 3 & 1 \\
\hline Chinese milk vetch & 10 & 20 days & 2800 & 3 & 1 \\
\hline Red bell pepper & 9 & 15 days & 2400 & 5 & \\
\hline Red clover & 20 & 18 days & 2640 & 6 & 5 \\
\hline Lettuce & 16 & 5 weeks & 5100 & 6 & 5 \\
\hline White clover & $0.18 \mathrm{~g}$ of seeds & 4 weeks & 4800 & 6 & $\mathrm{UI}^{b)}$ \\
\hline Pea & 7 & 3 weeks & 5300 & UI & 6 \\
\hline Morning glory & 4 & 3 weeks & 9460 & 6 & 5 , UI \\
\hline Sunflower & 2 & 14 days & 1850 & Heliolactone & UI \\
\hline Rice (cv. Nipponbare) & 6 & 4 weeks & 5490 & \multicolumn{2}{|c|}{2 and 5} \\
\hline Tobacco (cv. NA24) & 2 & 3 months & 12900 & \multicolumn{2}{|c|}{ 5, 4-hydroxy-1, UI } \\
\hline Tomato & 36 & 5 weeks & 309000 & \multicolumn{2}{|c|}{ UI } \\
\hline
\end{tabular}

${ }^{a)}$ Concentration rates of aquaculture water for LC-MS/MS analysis. ${ }^{b)}$ Unidentified SL. 

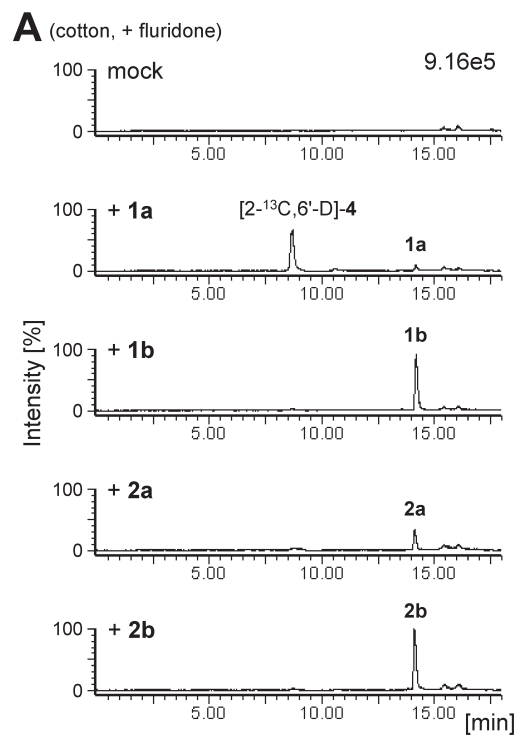

B
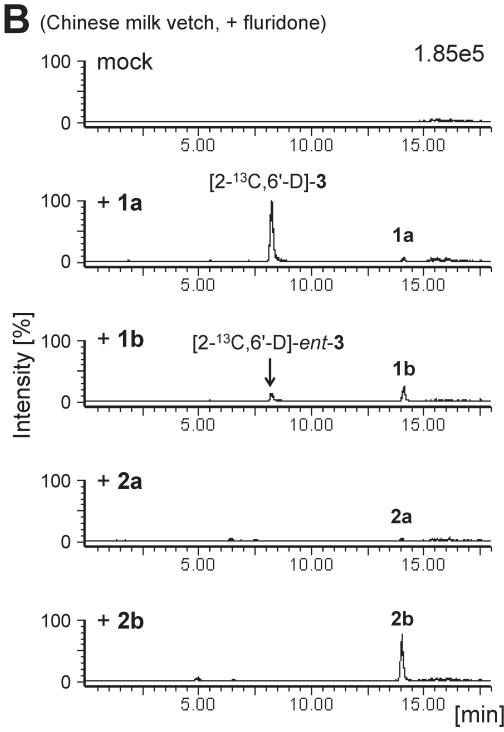

C (red bell pepper, - fluridone)
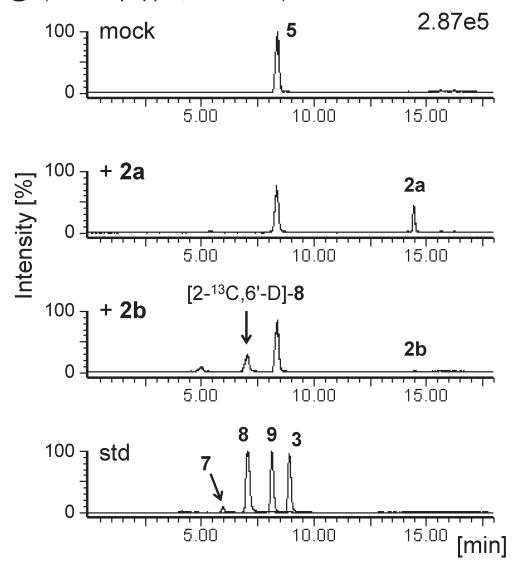

Fig. 2. Administration of $\left[2-{ }^{13} \mathrm{C}, 6{ }^{\prime}-\mathrm{D}\right]-5 \mathrm{DS}$ stereoisomers (1a, $\mathbf{1 b}, \mathbf{2} \mathbf{a}$, and $\left.\mathbf{2 b}\right)$ to the aquaculture water of cotton (A) and Chinese milk vetch (B) in the presence of fluridone $(0.4 \mu \mathrm{M})$ and to red bell pepper plants $(\mathrm{C})$ in the absence of fluridone. The intensity of the total ion current (TIC) chromatograms in MRM mode is normalized by the largest peak in all samples. std, authentic standards.

our experimental conditions. Table 1 summarizes the SLs detected under our experimental conditions.

As reported previously, ${ }^{21)}$ cotton (G. hirsutum) produced strigol (4) and 5DS (1), whereas G. arboreum produced sorgomol (3) and 5DS (1). The plant produced mainly HO-SLs, and the SL amounts were almost the same among the cotton cultivars. Chinese milk vetch produced mainly sorgomol (3) and a small amount of 5DS (1). Orobanchol (5) was not detected under our experimental conditions, although Yoneyama et al. ${ }^{26)}$ reported that the plant exuded not only 5DS (1) but also orobanchol (5) and alectrol (6). The red bell pepper plant only produced orobanchol (5). Red clover and lettuce plants produced orobanchol (5) and alectrol (6), as reported previously. ${ }^{18,27)}$ The major SL in these plants was alectrol (6). White clover and pea plants produced alectrol (6) and an unidentified SL that was detected by the channel at $m / z 405>97$, whose molecular weight was 16 mass units higher than that of alectrol (6). The unidentified SL was presumed to be fabacyl acetate because Xie et al. ${ }^{25)}$ and Foo and Davies ${ }^{28)}$ reported that the pea plant exuded fabacyl acetate in addition to orobanchol (5) and alectrol (6). The morning glory also produced alectrol (6) and its oxidized compound that was detected by the channel at $m / z 405>97$, but the chromatographic behavior of the oxidized compound differed from that in the pea and white clover. Known canonical SLs were not detected in sunflower root exudates even though we additionally analyzed the exudates with the parent ion scan at $m / z 97$, the fragment ion of the D ring, except for the non-tricyclic SL heliolactone, and an unidentified SL whose molecular weight was 376.9) The plants described above produced sufficient amounts of SLs for LC-MS/MS analysis in $24 \mathrm{hr}$ incubation. On the other hand, rice, tobacco, and tomato plants produced ca- nonical SLs, as reported previously, ${ }^{7)}$ but their amounts were insufficient for semi-quantification.

\section{Hydroxylation of 5DS stereoisomers by cotton and Chinese milk vetch}

Since we preliminary confirmed the hydroxylation of exogenous 5DS by the strigol-producing cotton (G. hirsutum) and Chinese milk vetch (A. sinicus) (Supplementary Fig. S1A and B), we administered stereoisomers of $\left[2-{ }^{13} \mathrm{C}, 6{ }^{\prime}-\mathrm{D}\right]-5 \mathrm{DS}$ (1a and $\mathbf{1 b}$ ) independently to aquacultures of cotton and Chinese milk vetch and allowed the plants to metabolize for $24 \mathrm{hr}$ in the presence of fluridone, an inhibitor of carotenoid biosynthesis, to reduce the production of endogenous SLs. Cotton specifically converted $\left[2-{ }^{13} \mathrm{C}, 6\right.$ '-D]-5DS (1a) into $\left[2-{ }^{13} \mathrm{C}, 6\right.$ ' $\left.-\mathrm{D}\right]$-strigol $\left(\left[2-{ }^{13} \mathrm{C}, 6^{\prime}-\mathrm{D}\right]-4\right)$, as reported by Iseki et al. ${ }^{21)}$ whereas the plant did not convert $\left[2-{ }^{13} \mathrm{C}, 6^{\prime}-\mathrm{D}\right]-$ ent-5DS (1b) into $\left[2-{ }^{13} \mathrm{C}, 6^{\prime}-\mathrm{D}\right]-$ ent-strigol ([2- $\left.{ }^{13} \mathrm{C}, 6{ }^{\prime}-\mathrm{D}\right]-$ ent-4) (Fig. $2 \mathrm{~A}$ and Supplementary Fig. S1C). The Chinese milk vetch also converted $\left[2-{ }^{13} \mathrm{C}, 6^{\prime}-\mathrm{D}\right]-$ 5DS (1a) into $\left[2-{ }^{13} \mathrm{C}, 66^{\prime}-\mathrm{D}\right]$-sorgomol $\left(\left[2-{ }^{13} \mathrm{C}, 66^{\prime}-\mathrm{D}\right]-3\right)$ (Fig. $2 \mathrm{~B}$ and Supplementary Fig. S1D). Unlike cotton, the Chinese milk vetch converted a trace amount of $\left[2-{ }^{13} \mathrm{C}, 6^{\prime}-\mathrm{D}\right]$-ent-5DS (1b) into $\left[2-{ }^{13} \mathrm{C}, 6^{\prime}-\mathrm{D}\right]$-ent-sorgomol $\left(\left[2-{ }^{13} \mathrm{C}, 66^{\prime}-\mathrm{D}\right]-\right.$-ent-3) as well as sorghum. ${ }^{11)}$ The conversions in these plants were dose-dependently inhibited by uniconazole-P, an inhibitor of cytochrome P450 (Supplementary Fig. S2). The half maximal (50\%) inhibitory concentration $\left(\mathrm{IC}_{50}\right)$ in cotton and Chinese milk vetch was $1 \mu \mathrm{M}$ and $0.5 \mu \mathrm{M}$, respectively. On the other hand, an inhibitor of 2-oxoglutarate-dependent dioxygenase, prohexadione, little inhibited the hydroxylation at $10 \mu \mathrm{M}$ (data not shown). Both plants negligibly metabolized $\left[2{ }^{13} \mathrm{C}, 6^{\prime}-\mathrm{D}\right]-4 \mathrm{DO}(\mathbf{2 a})$ and its enantiomer (2b) to remarkable HO-SLs (Fig. 2A, B and Supple- 
mentary Fig. S1C, D).

4. Hydroxylation of 4DO stereoisomers in the red bell pepper plant

In the preliminary experiment in which $r a c-d_{1}-4 D O\left(r a c-d_{1}-\right.$ $2)^{11)}$ was administered to the aquaculture water, the red bell pepper plant little converted the substrate into deuterium-labeled orobanchol (5) (Supplementary Fig. S3A). Instead, oxidized metabolites of $r a c-d_{1}-2$ were detected by the MRM channel at $\mathrm{m} / \mathrm{z}$ $348>97$ for $d_{1}$-HO-SLs. The major metabolite was also detected at the channel at $m / z 318>97$ for $d_{1}$-sorgomol $\left(d_{1}-3\right)$. Thus, we analyzed the authentic sorgomol isomers shown in Fig. 1C, 8-epi-sorgomol (7), 2',8-bisepi-sorgomol (8), and 2'-epi-sorgomol (9), ${ }^{11)}$ under the same conditions. The major metabolite at $t_{\mathrm{R}} 7.0$ min showed the same fragment pattern in the parent ion scan (Supplementary Fig. S3B) and the chromatographic behavior as those of $2^{\prime}, 8$-bisepi-sorgomol (8), which has the hydroxyl group at $\mathrm{C}-10$, the trans methyl carbon against the $\mathrm{C}$ ring (Supplementary Fig. S3A). To determine the structure of the metabolites, $\left[2-{ }^{13} \mathrm{C}, 6{ }^{\prime}-\mathrm{D}\right]-4 \mathrm{DO}(\mathbf{2 a})$ and its enantiomer $\left[2-{ }^{13} \mathrm{C}, 66^{\prime}-\mathrm{D}\right]-$ ent-4DO (2b) were administered to the aquaculture water of the red bell pepper plant in the absence of fluridone (Fig. 2C and Supplementary Fig. S3C). The major metabolite was detected in the root exudates administered with compound $\mathbf{2 b}$ after $48 \mathrm{hr}$. The production of the major metabolite was dose-dependently inhibited by uniconazole-P (data not shown). The minor metabolite of $\mathbf{2 b}$ at $t_{\mathrm{R}} 5.0 \mathrm{~min}$ was detected in the transition at $\mathrm{m} / \mathrm{z}$ $349>97$ but not at $m / z$ 319>97 (Supplementary Fig. S3C). The chromatographic behavior of the minor metabolite was consistent with an isomer of 7-hydroxy-5DS (10), but their configuration was unclear because the 7-hydroxy-5DS (10) isomers were indistinct under our analytical conditions. A small amount of $\left[2-{ }^{13} \mathrm{C}, 6\right.$ ' $\left.-\mathrm{D}\right]-4 \mathrm{DO}(\mathbf{2 a})$ was converted into ent-2' -epi-sorgomol (ent-9) (Supplementary Fig. S3C) and an unknown hydroxylated compound at $t_{\mathrm{R}} 5.5 \mathrm{~min}$. The red bell pepper converted trace amounts of rac- $d_{1}-5 \mathrm{DS}\left(\mathrm{rac}-d_{1}-1\right)$ into oxygenated metabolites, whose structures were unknown (Supplementary Fig. S3A).

\section{Hydroxylation of 4DO stereoisomers by orobanchol-producing plants}

To investigate whether other orobanchol-producing plants can convert 4DO (2) into orobanchol (5), we administered stable isotope-labeled $4 \mathrm{DO}$ stereoisomers $\left(r a c-d_{1}-\mathbf{2}, \mathbf{2 a}\right.$, and $\left.\mathbf{2 b}\right)$ to hydroponically cultured rice, red clover, and pea plants in the absence of fluridone. The conversion was evaluated via LC-MS/ MS analyses using MRM with channels for $\mathrm{M}+1 / \mathrm{M}+2$ of HO-SLs. The rice plant converted $r a c-d_{1}-4 \mathrm{DO}\left(r a c-d_{1}-2\right)$ into $d_{1}$-orobanchol $\left(d_{1}-5\right)$, as reported by Zhang et al. ${ }^{20)}$ (Supplementary Fig. S4A). The amount of $d_{1}-\mathbf{5}$ was too small to semiquantify by peak area. In red clover, which produces mainly alectrol (6) as well as orobanchol (5) as a minor SL, the area of $\mathrm{M}+1$ in orobanchol was slightly increased by the administration of $r a c-d_{1}-2$ (Supplementary Fig. S4B). The isotopic abundance ratio of $\mathrm{M}+1$ in orobanchol increased to $23 \%$ due to the

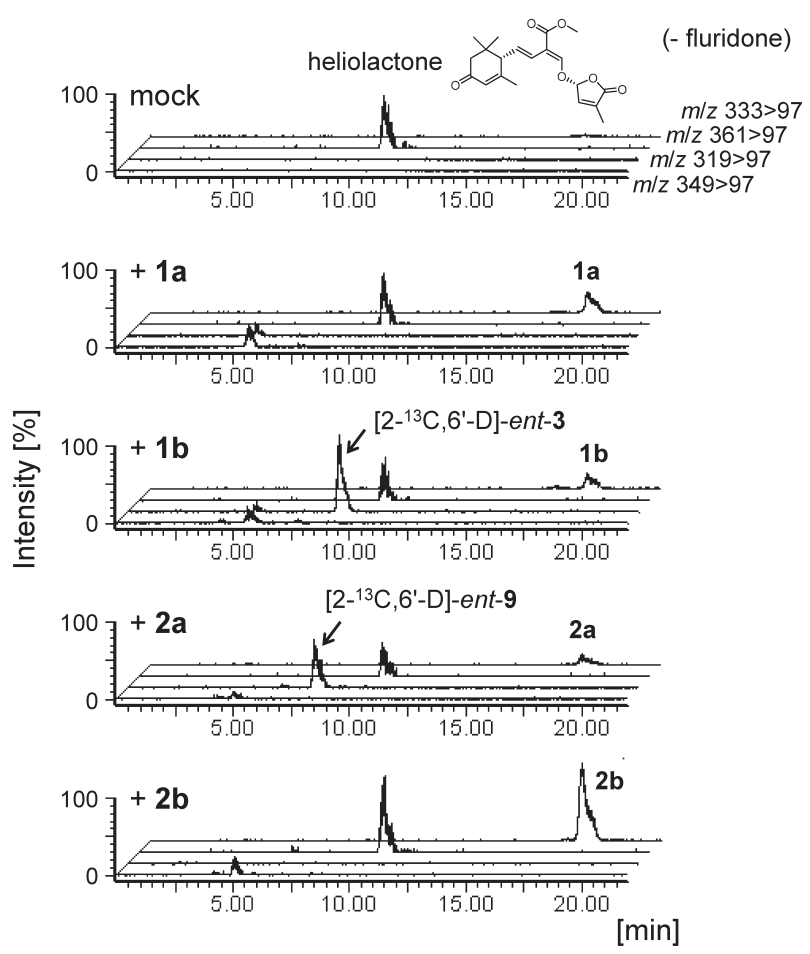

Fig. 3. MRM chromatograms of the aquaculture water of the sunflower administered with $\left[2-{ }^{13} \mathrm{C}, 6{ }^{\prime}-\mathrm{D}\right]-5 \mathrm{DS}$ stereoisomers (1a, 1b, 2a, and $\mathbf{2 b}$ ) in the absence of fluridone. The MRM transitions selected are at $m / z 333>97$ for compounds $\mathbf{1 a}, \mathbf{1 b}, \mathbf{2} \mathbf{a}$, and $\mathbf{2} \mathbf{b}$; at $\mathrm{m} / z \mathbf{3 6 1}>97$ for heliolactone; at $\mathrm{m} / \mathrm{z}$ $319>97$ for labeled sorgomol isomers; and at $\mathrm{m} / z$ 349>97 for labeled HOSLs. The ion intensities of channels at $\mathrm{m} / z 333>97,361>97,319>97$, and $349>97$ are $1.26 \mathrm{e} 5,1.53 \mathrm{e} 4,3.67 \mathrm{e} 4$, and $3.67 \mathrm{e} 4$, respectively.

administration of rac- $d_{1}-2$, as compared to $15 \%$ in the control, but the ratio showed no significant difference against the control $(p=0.12, n=3)$. The area of the $M+1$ peak of alectrol (6) did not increase upon administration of the deuterium-labeled substrate. We also detected deuterium-labeled 2',8-bisepisorgomol $\left(d_{1}-8\right)$ in the root exudates of red clover administered with $r a c-d_{1}-2$. On the other hand, identifiable metabolites including $\left[2-{ }^{13} \mathrm{C}, 6^{\prime}-\mathrm{D}\right]$-orobanchol $\left(\left[2-{ }^{13} \mathrm{C}, 66^{\prime}-\mathrm{D}\right]-5\right)$ and $\left[2-{ }^{13} \mathrm{C}, 66^{\prime}-\mathrm{D}\right]$-sorgomol isomers $\left(\left[2-{ }^{13} \mathrm{C}, 6^{\prime}-\mathrm{D}\right]-\mathbf{3},-7,-\mathbf{8}\right.$, and $\mathbf{- 9}$ and their enantiomers) were not detected from the root exudates of pea plants administered with labeled substrates $2 \mathbf{a}$ and 2b (Supplementary Fig. S4C).

\section{Hydroxylation of 5DS stereoisomers by the sunflower}

We administered isotope-labeled 5DS stereoisomers (1a, 1b, $\mathbf{2 a}$, and $\mathbf{2 b}$ ) without fluridone to the sunflower, which does not produce canonical tricyclic SLs, to confirm the hydroxylation of the methyl groups in the A ring. The sunflower converted $\left[2-{ }^{13} \mathrm{C}, 6^{\prime}-\mathrm{D}\right]-e n t-5 \mathrm{DS}(\mathbf{1 b})$ and $\left[2-{ }^{13} \mathrm{C}, 6^{\prime}-\mathrm{D}\right]-4 \mathrm{DO}$ (2a) into $\left[2-{ }^{13} \mathrm{C}, 6^{\prime}-\mathrm{D}\right]$-ent-sorgomol $\left(\left[2-{ }^{13} \mathrm{C}, 6^{\prime}-\mathrm{D}\right]-\right.$ ent-3) and $\left[2-{ }^{13} \mathrm{C}, 6{ }^{\prime}-\mathrm{D}\right]$-ent-2' ${ }^{\prime}$-epi-sorgomol $\left(\left[2-{ }^{13} \mathrm{C}, 6{ }^{\prime}-\mathrm{D}\right]\right.$-ent-9), respectively (Fig. 3). All substrates were converted into the hydroxylated metabolites that were presumed to be 7-hydroxy-5DS (10), but the amounts were small. The production of endogenous he- 
liolactone was negligibly inhibited by the administration of labeled substrates.

\section{Discussion}

In this research, we confirmed the hydroxylation of deoxy-SLs by seven plants. We additionally demonstrated the stereoselective conversion of 5DS (1) into strigol (4) and sorgomol (3) by cotton and Chinese milk vetch, respectively (Fig. 2A, B). The hydroxylation reactions were inhibited by treatment of uniconazole-P, which indicates that cytochrome P450 enzymes are involved in the introduction of the hydroxyl group to 5DS (1), as well as sorghum. ${ }^{11)}$ We also confirmed the conversion of $4 \mathrm{DO}$ (2) into orobanchol (5) in rice, as reported by Zhang et al. ${ }^{20)}$ (Supplementary Fig. S4A). However, the red bell pepper, red clover, and pea plants failed to convert 4DO (2) into orobanchol (5), as well as the cowpea ${ }^{21)}$ (Fig. 2C and Supplementary Figs. S3C, S4B, C). Since unmetabolized substrates were detected in red bell pepper and red clover roots (data not shown), it is unlikely that most of the substrates were degraded before conversion into orobanchol (5) in the administration experiments. Actually, these plants converted exogenous isotope-labeled 4DO (2) into $2^{\prime}, 8$-bisepi-sorgomol (8). A small amount of exogenous $4 \mathrm{DO}$ (2) was converted into ent-2'-epi-sorgomol (ent-9) in the red bell pepper plant (Supplementary Fig. S3C). This result indicates that the plants have the ability to oxidize the exogenous substrates.

In addition to the cowpea, ${ }^{21)}$ red bell pepper, red clover, and pea plants seem to produce orobanchol (5) directly, without passing through $4 \mathrm{DO}(2)$. This is explained by the fact that $4 \mathrm{DO}$ (2) was negligibly detected in the root exudates of these plants in our experimental conditions. In contrast, 4DO (2) was detected in rice root exudates (Table 1). 5DS (1) was also detected in the root exudates of cotton and Chinese milk vetch, which converted exogenous 5DS (1) into the hydroxylated metabolite corresponding to the endogenous HO-SLs. Detecting 5DS (1) or 4DO (2) can be hint as to whether plants are able to convert deoxySLs into HO-SLs. Orobanchol-producing plants are not only the red bell pepper and red clover but also burdock (Arctium lappa L.) and the soybean (Glycine $\max \left(\right.$ L.) Merrill). ${ }^{7)}$ Since burdock and soybean plants are reported to produce 5DS (stereoisomers) in the review, these plants might convert 4DO (2) to orobanchol (5). Additional feeding experiments are required to demonstrate the biosynthetic pathway of orobanchol (5) via 4DO (2).

Orobanchol (5) in the red bell pepper is probably produced from carlactone (CL), as with the cowpea, ${ }^{21)}$ because CL was detected in root extracts of the red bell pepper and cowpea (data not shown). The oxidations of CL to produce bioactive SLs were catalyzed by at least a cytochrome P450 enzyme CYP711.20,29) Differences of enzymatic reaction mechanisms among CYP711 isoforms may cause the diversity in the biosynthetic pathway of orobanchol (5). A rice CYP711 enzyme Os900 (CYP711A2) converted CL into 4DO (2), and a second rice CYP711 isoform Os1400 (CYP711A3) converted 4DO (2) into orobanchol (5). ${ }^{20)}$ On the other hand, Arabidopsis CYP711 (CYP711A1)

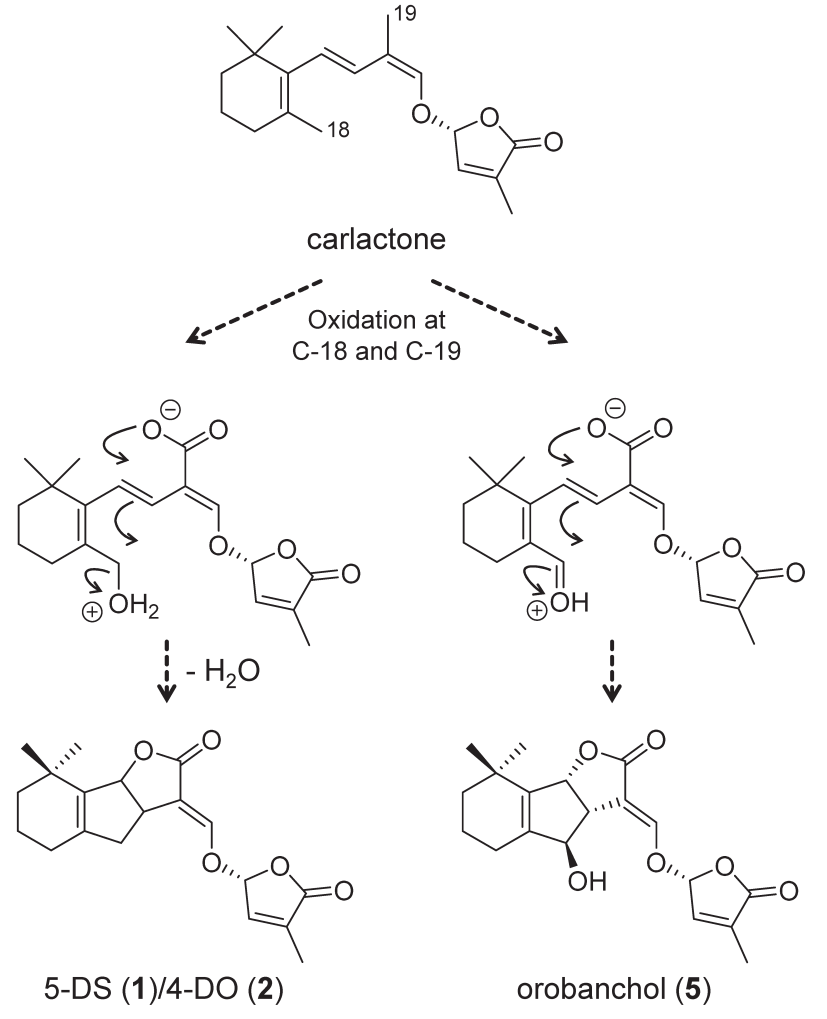

Fig. 4. Proposal BC rings formation of tricyclic SLs.

converted CL into carlactonoic acid (CLA). In Arabidopsis, a 2-oxoglutarate- and Fe(II)-dependent dioxygenase, LATERAL BRANCHING OXIDOREDUCTASE (LBO), is involved in the production of bioactive SLs. ${ }^{30}$ The enzyme functions in the final stages of active SL biosynthesis, although the enzymatic detail is unknown. CYP711A isoforms in red bell pepper, red clover, and pea plants may directly convert CL into orobanchol (5) or may convert CLA into orobanchol (5) with other oxygenases such as LBO after introducing the carboxyl group to CL by CYP711. The oxidation of $\mathrm{C}-18$, the methyl group in the six-membered ring of $\mathrm{CL}$, is likely to be required to form the $\mathrm{B}$ ring, as the oxidation of $\mathrm{C}-19$ is required to form the $\mathrm{C}$ ring $^{20,31}$ ) (Fig. 4). In the Bring formation of 5DS (1) and 4DO (2), the protonated hydroxyl group at C-18 will act as a leaving group, but if the methyl group at $\mathrm{C}-18$ was converted to an aldehyde group, the oxygen would remain in the structure as a hydroxyl group on the $\mathrm{B}$ ring to give orobanchol (5). Indeed, an oxatricyclic compound having the hydroxy-cyclopentene framework was generated from a hydroxylated cis-2,4-dien-1-al fused with a six-membered ring by a gold catalyst. ${ }^{32)}$ The oxidation at C-18 of CL could occur prior to carboxylation at C-19. Further research, such as administration experiments and structural analyses of oxygenases, is needed for elucidation of the direct synthetic pathway of orobanchol (5).

One of the hydroxylated products of exogenous deoxy-SL in red bell pepper and red clover plants, $2^{\prime}, 8$-bisepi-sorgomol (8), has not yet been found as a naturally occurring SL in the root exudates of any plants. The conversion of ent-4DO (ent-2) into 
$2^{\prime}, 8$-bisepi-sorgomol (8) was not observed in pea plants (Supplementary Fig. S4C). The reaction might be limited in some plant species. The production of $2^{\prime}, 8$-bisepi-sorgomol (8) was inhibited by uniconazole-P, a cytochrome $\mathrm{P} 450$ inhibitor, in red bell pepper plants. The cytochrome $\mathrm{P} 450$ enzymes are involved in the detoxification of xenobiotics, such as herbicides, in many plants. ${ }^{33,34)}$ The hydroxylation of $\left[2-{ }^{13} \mathrm{C}, 6^{\prime}-\mathrm{D}\right]$-ent-4DO (2b) is probably for xenobiotic metabolism in red bell pepper plants. Similarly, hydroxylation of the methyl group of $\left[2-{ }^{13} \mathrm{C}, 6^{\prime}-\mathrm{D}\right]-$ ent-5DS (1b) and $\left[2-{ }^{13} \mathrm{C}, 6{ }^{\prime}-\mathrm{D}\right]-4 \mathrm{DO}(\mathbf{2 a})$ by the sunflower (Fig. 3 ) will decrease the activities of exogenous SLs, since the activities of HO-SLs were lower than those of deoxy-SLs on the inhibition of shoot branching in rice ${ }^{35)}$ and the induction of seed germination against Striga hermonthica. ${ }^{36)}$ The production of endogenous SL was little inhibited by the administration of deoxySLs in both the red bell pepper and the sunflower. This result implies the possibility that hydroxylation of exogenous deoxySLs is a metabolic reaction distinct from endogenous SL biosynthesis.

In conclusion, we reconfirmed the conversion of 5DS (1) into strigol (4) and sorgomol (3). However, the conversion of $4 \mathrm{DO}$ (2) into orobanchol (5) was not confirmed in red bell pepper, red clover, or pea plants under our experimental conditions, although rice plants converted exogenous 4DO (2) into orobanchol (5). The results suggest the diversity of biosynthetic pathways of canonical hydroxylated SLs: introduction of the hydroxyl group into 5DS (1) or 4DO (2), and direct conversion to orobanchol (2). We have to consider the metabolism of CL, a precursor of SLs, including the indication for the direct synthetic pathway of orobanchol (5). Collaboration in the fields of molecular biology, analytical chemistry, and natural product chemistry is required to elucidate the biosynthetic pathway of SLs. The identification of enzymes involved in SL biosynthesis will help to develop specific inhibitors for SL biosynthesis and hydroxylation. Using appropriate inhibitors for the enzymes might temporarily and quantitatively change the SL contents in root exudates, which would facilitate the control of weeds parasitic to the roots of host crops.

\section{Acknowledgements}

This work was supported, in part, by grants from JST/JICA, Science and Technology Research Partnership for Sustainable Development (SATREPS) and JSPS KAKENHI Grant numbers 11J1777, 23580149, 24658111, 25292065, and 26850071.

\section{References}

1) K. Akiyama, K. Matsuzaki and H. Hayashi: Nature 435, 824-827 (2005).

2) X. Xie, K. Yoneyama and K. Yoneyama: Annu. Rev. Phytopathol. 48, 93-117 (2010).

3) C. Ruyter-Spira, S. Al-Babilli, S. van der Krol and H. Bouwmeester: Trends Plant Sci. 18, 72-83 (2013).

4) V. Gomez-Roldan, S. Fermas, P. B. Brewer, V. Puech-Pages, E. A. Dun, J. P. Pillot, F. Letisse, R. Matusova, S. Danoun, J. C. Portais, H. Bouwmeester, G. Bécard, C. A. Beveridge, C. Rameau and S. F. Ro- change: Nature 455, 189-194 (2008).

5) M. Umehara, A. Hanada, S. Yoshida, K. Akiyama, T. Arite, N. Takeda-Kamiya, H. Magome, Y. Kamiya, K. Shirasu, K. Yoneyama, J. Kyozuka and S. Yamaguchi: Nature 455, 195-200 (2008).

6) P.-M. Delaux, X. Xie, R. E. Timme, V. Puech-Pages, C. Dunand, E. Lecompte, C. F. Delwiche, K. Yoneyama, G. Bécard and N. SéjalonDelmas: New Phytol. 195, 857-871 (2012).

7) S. Ćavar, B. Zwanenburg and P. Tarkowski: Phytochem. Rev. 14, 691711 (2015).

8) H. I. Kim, T. Kisugi, P. Khetkam, X. Xie, K. Yoneyama, K. Uchida, T. Yokota, T. Nomura, C. S. P. McErlean and K. Yoneyama: Phytochemistry 103, 85-88 (2014).

9) K. Ueno, T. Furumoto, S. Umeda, M. Mizutani, H. Takikawa, R. Batchvarova and Y. Sugimoto: Phytochemistry 108, 122-128 (2014).

10) M. Jamil, J. Rodenburg, T. Charnikhova and H. J. Bouwmeester: New Phytol. 192, 964-975 (2011).

11) N. Motonami, K. Ueno, H. Nakashima, S. Nomura, M. Mizutani, H. Takikawa and Y. Sugimoto: Phytochemistry 93, 41-48 (2013).

12) H. Koltai, S. P. LekKala, C. Bhattacharya, E. Mayzlish-Gati, N. Resnick, S. Wininger, E. Dor, K. Yoneyama, K. Yoneyama, J. Hershenhorn, D. M. Joel and Y. Kapulnik: J. Exp. Bot. 61, 1739-1749 (2010).

13) K. Yoneyama, A. A. Awad, X. Xie, K. Yoneyama and Y. Takeuchi: Plant Cell Physiol. 51, 1095-1103 (2010).

14) K. Yoneyama, R. Arakawa, K. Ishimoto, H. I. Kim, T. Kisugi, X. Xie, T. Nomura, F. Kanampiu, T. Yokota, T. Ezawa and K. Yoneyama: New Physiol. 206, 983-989 (2015).

15) C. E. Cook, L. P. Whichard, B. Turner, M. E. Wall and G. H. Egley: Science 154, 1189-1190 (1966).

16) X. Xie, K. Yoneyama, D. Kusumoto, Y. Yamada, Y. Takeuchi, Y. Sugimoto and K. Yoneyama: Tetrah Lett. 49, 2066-2068 (2008).

17) S. Müller, C. Hauck and H. Schildknecht: J. Plant Growth Regul. 11, 77-84 (1992).

18) K. Ueno, S. Nomura, S. Muranaka, M. Mizutani, H. Takikawa and Y. Sugimoto: J. Agric. Food Chem. 59, 10485-10490 (2011).

19) T. Yokota, H. Sakai, K. Okuno, K. Yoneyama and Y. Takeuchi: Phytochemistry 49, 1967-1973 (1998).

20) Y. Zhang, A. D. van Dijk, A. Scaffidi, G. R. Flematti, M. Hofmann, T. Charnikhova, F. Verstappen, J. Hepworth, S. van der Krol, O. Leyser, S. M. Smith, B. Zwanenburg, S. Al-Babili, C. Ruyter-Spira and H. J. Bouwmeester: Nat. Chem. Biol. 10, 1028-1033 (2014).

21) M. Iseki, K. Shida, K. Kuwabara, T. Wakabayashi, M. Mizutani, H. Takikawa and Y. Sugimoto: J. Exp. Bot. 69, 2305-2318 (2018).

22) M. Tanaka, Y. Sugimoto, M. Kuse and H. Takikawa: Biosci. Biotechnol. Biochem. 77, 832-835 (2013).

23) K. Ueno, A. Hanada, S. Yamaguchi and T. Asami: J. Labelled Comp. Radiopharm. 53, 763-766 (2010).

24) X. Xie, D. Kusumoto, Y. Takeuchi, K. Yoneyama, Y. Yamada and K. Yoneyama: J. Agric. Food Chem. 55, 8067-8072 (2007).

25) X. Xie, K. Yoneyama, Y. Harada, N. Fusegi, Y. Yamada, S. Ito, T. Yokota, Y. Takeuchi and K. Yoneyama: Phytochemistry 70, 211-215 (2009).

26) K. Yoneyama, X. Xie, H. Sekimoto, Y. Takeuchi, S. Ogasawara, K. Akiyama, H. Hayashi and K. Yoneyama: New Phytol. 179, 484-494 (2008).

27) K. Yoneyama, X. Xie, T. Kisugi, T. Nomura, H. Sekimoto, T. Yokota and K. Yoneyama: Plant Growth Regul. 65, 495-504 (2011).

28) E. Foo and N. W. Davies: Planta 234, 1073-1081 (2011).

29) S. Abe, A. Sado, K. Tanaka, T. Kisugi, K. Asami, S. Ota, H. I. Kim, K. Yoneyama, X. Xie, T. Ohnishi, Y. Seto, S. Yamaguchi, K. Akiyama, K. Yoneyama and T. Nomura: Proc. Natl. Acad. Sci. U.S.A. 111, 1808418089 (2014). 
30) P. B. Brewer, K. Yoneyama, F. Filardo, E. Meyers, A. Scaffidi, T. Frickey, K. Akiyama, Y. Seto, E. A. Dun, J. E. Cremer, S. C. Kerr, M. T. Waters, G. R. Flematti, M. G. Mason, G. Weiller, S. Yamaguchi, T. Nomura, S. M. Smith, K. Yoneyama and C. A. Beveridge: Proc. Natl. Acad. Sci. U.S.A. 113, 6301-6306 (2016).

31) B. Zwanenburg, S. Ćavar Zeljković and T. Pospíšil: Pest Manag. Sci. 72, 15-29 (2016).

32) C. C. Lin, T. M. Teng, A. Odedra and R. S. Liu: J. Am. Chem. Soc. 129, 3798-3799 (2007).
33) M. A. Schuler: Crit. Rev. Plant Sci. 15, 235-284 (1996).

34) T. Robineau, Y. Batard, S. Nedelkina, F. Cabello-Hurtado, M. LeRet, O. Sorokine, L. Didierjean and D. Werck-Reichhart: Plant Physiol. 118, 1049-1056 (1998).

35) M. Umehara, M. Cao, K. Akiyama, T. Akatsu, Y. Seto, A. Hanada, W. Li, N. Takeda-Kamiya, Y. Morimoto and S. Yamaguchi: Plant Cell Physiol. 56, 1059-1072 (2015).

36) K. Ueno, S. Ishiwa, H. Nakashima, M. Mizutani, H. Takikawa and Y. Sugimoto: Bioorg. Med. Chem. 23, 6100-6110 (2015). 